\title{
Characteristics of immune responses to three coronavirus infections: SARS, MERS and SARS-CoV-2
}

\author{
Weilai Ye \\ Shanghai Foreign Language School affiliated to SISU, Shanghai, China
}

Keywords: coronavirus; epidemiology; infection; immune response.

\begin{abstract}
The outbreaks of coronavirus have posed threats to the world several times in decades. The immune responses are crucial during the coronavirus infection. Studying immune responses will contribute to the development of target therapy and vaccines. This review provides a brief introduction to the epidemiology of three coronaviruses, differences in infections, and the corresponding immune responses.
\end{abstract}

\section{Introduction}

At the end of 2019, the outbreak of a coronavirus called COVID-19 was reported in Wuhan, Hubei, China. Unfortunately, two coronavirus pandemics have threatened the world, Severe Acute Respiratory Syndrome (SARS) in 2002-2003 and Middle East Respiratory Syndrome (MERS) in 2011 [1]. SARS-CoV, MERS-CoV and SARS-CoV-2 have similar, but various genome composition [1]. Coronaviruses are distributed widely among mammals and birdsand cause mainly respiratory or enteric diseases [2]. Coronaviruses are usually highly species-specific to their corresponding receptors, while the adaption of several coronaviruses to human proved that they are also mutable [3]. Studies have been done to figure out the structure, infection mechanism and immune responses induced by SARS-CoV, MERS-CoV and SARS-CoV-2. This review narrates and compares the epidemiology and immune responses induced by the three coronaviruses.

\section{Epidemiology of coronavirus}

\subsection{Coronavirus Structure}

Coronaviruses have the largest genomes for RNA viruses [4], which are enveloped, single-stranded, positive-sense RNA viruses [5], and belong to the family Coronaviridae, the subfamily Corona-virinae and the order Nidovirales [6]. The spike (S) protein, membrane (M) protein, envelope $(\mathrm{E})$ protein and nucleocapsid $(\mathrm{N})$ protein are structural proteins that are needed to form a complete virus particle (figure 1) [3]. The more variable region of the spike molecule, S1, is the part that binds to the receptor, which leads to the structural change of S2, mediating fusion between the virus and cell membranes [3]. Interactions between the $S$ protein and its receptor initiate the attachment of the virus to the host cell [7]. SARS-CoV and SARS-CoV-2 use angiotensin-converting enzyme 2 (ACE2) as a receptor, which is expressed on the epithelial cells of alveoli, trachea, and bronchi, bronchial serous glands, and alveolar monocytes and macrophages [8], while MERS-CoV uses dipeptidyl-peptidase 4 (DPP4) [9], which is a multifunctional cell-surface protein widely expressed on epithelial cells in the kidney, small intestine, liver and prostate and on activated leukocytes [8].

\subsection{Differences in coronavirus infection}

\subsubsection{SARS}

SARS virus mainly binds to the receptor ACE2, which is a surface molecule on arterial and venous endothelial cells, arterial smooth muscle cells, epithelia of the small intestine, and the respiratory tract [8]. In the respiratory tract, ACE2 is expressed on the epithelial cells of alveoli, 
trachea, and bronchi, bronchial serous glands, and alveolar monocytes and macrophages [8]. The down degradation of ACE2 leads to the pathological changes in the lung [10,11].

\subsubsection{MERS}

The first step of infection for the MRES virus is binding to the receptor Dipeptidyl peptidase 4 [8,12]. It is a multifunctional cell-surface protein widely expressed on epithelial cells in the kidney, small intestine, liver and prostate and on activated leukocytes, which is mainly in the alveoli in the human respiratory tract [12]. It is also found that MERS-CoV can infect human immune cells, including dendritic cells, macrophages, and T cells [13,14,15].

\subsubsection{SARS-CoV-2}

Based on the published article and clinical observations of COVID-19 patients, it is proposed that the virus might pass through the mucous membranes, especially nasal and larynx mucosa, and then enters the lungs through the respiratory tract [16]. The virus might enter the peripheral blood from the lungs, which causes viremia. It would attack the targeting organs that express ACE2, such as the lungs, heart, renal, gastrointestinal tract $[17,18]$. Unlike SARS-CoV, SARS-CoV-2 S proteins could be triggered upon the receptor binding without exogenous protease priming or activation [19]. Experiments indicated that SARS-CoV-2's S pseudovirions enter 293/hACE2 cells mainly through endocytosis [19]. Meanwhile, PIKfyve and TPC2 are important for entry [19].

\section{Immune response induced by coronavirus}

\subsection{Cellular immunity}

For all we knew, T cell plays an important role in antiviral immunity. Recently blood samples were collected from 46 cases of SARS patients, compared with normal controls, the cell count of naive CD4+T cell subset was remarkably decreased, and the percentage of CD8+CD38+T cell subset was higher during the 1st week. While during the 3rd-5th week, the CD8+T cell count and the percentage of CD8+CD38+T cell subset reached normal values [20]. However, during the 8th-12th week, the cell counts of CD4+T cell and naive CD4+T cell subset was still less than those of normal controls [20]. And the higher frequency of poly-functional CD4+T cells and CD8+T cells are detected in the severe group [1]. Distinctively high frequencies of MERS coronavirus-reactive CD8+ $\mathrm{T}$ cells were observed in patients with severe/moderate illness, while antibody and CD4+ T-cell responses were minimally detected at this stage [21]. And lymphopenia was observed in both SARS-CoV and SARS- CoV-2 infections [22]. CD8+ T cells responded preferentially to the viral S protein compared with $\mathrm{E} / \mathrm{M} / \mathrm{N}$ proteins, especially at the acute stage, slightly more CD4+ $\mathrm{T}$ cells recognized $\mathrm{E} / \mathrm{M} / \mathrm{N}$ proteins compared with $\mathrm{S}$ protein at the convalescent phase [21]. The difference in CD4:CD8 ratio, CD4+T cell frequency, CD8+T cell frequency and CD4+ T cell's mean fluorescence intensity (MFI) was not significant between COVID-19 patients and healthy individuals, while the CD8+T cell's MFI increased significantly in COVID-19 infected patients [23].

\subsection{Humoral immunity}

In humoral immunity, IgG against $\mathrm{N}$ protein can be detected in the sera as early as 4 days after SARS illness onset, with most patients seroconverting by day 14 [24]. Neutralizing IgG is major in the neutralization of the SARS-CoV [22]. And long-lasting specific IgG and neutralizing antibody are reported as long as 2 years after infection [25]. For MERS-CoV infection, seroconversion is seen in the second or third week of disease onset [1]. While IgM, IgA, and IgG antibodies against SARS-CoV-2 appear day 1 after the symptom onset [26]. The IgM and IgA antibodies were both detectable on day 5, and the median time of appearance of IgG was on day 14 [26]. The median seroconversion time for total antibodies, IgM and then IgG were day-11, day-12 and day-14 [27]. 


\subsection{Cytokines}

SARS-CoV infection of dendritic cells induces low-level expression of antiviral cytokines IFN- $\alpha \beta$, moderate up-regulation of pro-inflammatory cytokines TNF and IL-6, and a significant up-regulation of inflammatory chemokines CCL3, CCL5, CCL2, and CXCL10 [28,29]. And the infection of macrophages induces delayed but elevated levels of IFN and other pro-inflammatory cytokines [29]. The SARS-CoV-infected airway epithelial cells (AECs) also produce large amounts of CCL3, CCL5, CCL2, and CXCL10 [30]. While MERS-CoV infection of human airway epithelial cells also induces significant but delayed IFN and pro-inflammatory cytokine (IL-1 $\beta$, IL-6, and IL-8) responses [31]. MERS-CoV replicates both in naïve and activated human monocyte-macrophages and DCs, but only activated $\mathrm{T}$ cells support MERS-CoV replication [32,33,34], which is different from SARS-CoV. MERS-CoV infection of THP-1 cells, a monocyte cell line, and human peripheral blood monocyte-derived macrophages and dendritic cells induced delayed but elevated levels of pro-inflammatory cytokines and chemokines such as CCL-2, CCL-3, CCL-5, IL-2, and IL-8 [33,34]. T cell immunity-associated cytokines IL-12 and IFN-g levels were lower in a fatal case compared to a patient who survived the MERS infection [35]. High levels of pro-inflammatory cytokines including IL-2, IL-7, IL-10, G-CSF, IP-10, MCP-1, MIP-1A, and TNF $\alpha$ were observed in 41 COVID-19 severe cases [36]. This early high rise in the serum levels of pro-inflammatory cytokines was also observed in SARS-CoV and MERS-CoV infection, which means there is a potential similar cytokine storm-mediated disease severity $[37,38]$.

\section{Conclusion}

Immune responses are important during coronavirus infections. The similarities and differences of immune responses induced by SARS-CoV, MERS-CoV, and SARS-CoV-2 are presented in this review. During SARS-CoV infection, the percentage of naive CD4+ subset decreases, and the percentage of CD8+CD38+ subset increases. For MERS-CoV and SARS-CoV-2 infections, CD8+T cell's mean fluorescence intensity(MFI) is higher compared to healthy individuals [20]. And antibodies including IgG, IgM and IgA can be detected during infections [24, 25, 26]. Therefore, exploring and comparing the human immune response caused by three different coronavirus infections are helpful to immunological methods that prevent and treat coronavirus pandemics.

\section{References}

[1] Eakachai Prompetchara, Chutitorn Ketloy, Tanapat Palaga. Immune responses in COVID-19 and potential vaccines: Lessons learned from SARS and MERS epidemic. Asian Pac J Allergy Immunol DOI 10.12932/AP-200220-0772

[2] Lai M.M.C., Holmes K.V. Coronaviridae: The viruses and their replication. In: Knipe D.M., Howley P.M., editors. "Fields Virology”. 4th edn. Lippincott, Williams \& Wilkins; Philadelphia: 2001. pp. 1163-1185.

[3] Masters PS. The molecular biology of coronaviruses. Adv Virus Res. 2006;66:193-292.

[4] Schoeman D, Fielding BC. Coronavirus envelope protein: current knowledge. Virol J. 2019;16(1):69. Published 2019 May 27.

[5] Szczepanski A, Owczarek K, Bzowska M, et al. Canine Respiratory Coronavirus, Bovine Coronavirus, and Human Coronavirus OC43: Receptors and Attachment Factors. Viruses. 2019;11(4):328. Published 2019 Apr 5.

[6] Cui J, Li F, Shi ZL. Origin and evolution of pathogenic coronaviruses. Nat Rev Microbiol. 2019;17(3):181-192.

[7] Fehr AR, Perlman S. Coronaviruses: an overview of their replication and pathogenesis. Methods Mol Biol. 2015; 1282:1-23. 
[8] Yin Y, Wunderink RG. MERS, SARS and other coronaviruses as causes of pneumonia. Respirology. 2018;23(2):130-137.

[9] Zhou P, Yang XL, Wang XG, et al. A pneumonia outbreak associated with a new coronavirus of probable bat origin. Nature [Preprint]. 2020 [cited 2020 Feb 15]: [15 p.]. Available from: https://doi. org/10.1038/s41586-020-2012-7

[10] Imai Y, Kuba K, Ohto-Nakanishi T, Penninger JM. Angiotensin-converting enzyme 2 (ACE2) in disease pathogenesis. Circ. J. 2010; 74: 405-10.

[11] Kuba K, Imai Y, Rao S, et al. A crucial role of angiotensin converting enzyme 2 (ACE2) in SARS coronavirus-induced lung injury. Nat. Med. 2005; 11: 875-9.

[12] Meyerholz DK, Lambertz AM, PB MC Jr. Dipeptidyl peptidase 4 distribution in the human respiratory tract: implications for the Middle East respiratory syndrome. Am J Pathol. 2016; 186: 78-86.

[13] Chu H, Zhou J, Ho-Yin wong B, et al. Productive replication of Middle East respiratory syndrome coronavirus in monocyte-derived dendritic cells modulates innate immune response. Virology. 2014;454-455: 197-205.

[14] Zhou J, Chu H, Li C, et al. Active replication of Middle East respiratory syndrome coronavirus and aberrant induction of inflammatory cyto- kines and chemokines in human macrophages: implications for pa- thogenesis. J Infect Dis. 2014;209(9):1331-1342.

[15] Chu H, Zhou J, Wong BHY, et al. Middle East respiratory syndrome coronavirus efficiently infects human primary $\mathrm{T}$ lymphocytes and activates the extrinsic and intrinsic apoptosis pathways. $\mathrm{J}$ Infect Dis. 2016;213(6):904-914.

[16] Lin L, Lu L, Cao W, Li T. Hypothesis for potential pathogenesis of SARS-CoV-2 infection-a review of immune changes in patients with viral pneumonia. Emerg Microbes Infect. 2020;9(1):727-732.

[17] Dan H, Maureen G, Richard B, et al. Quantitative mRNA expression prođling of ACE 2, a novel homol- ogue of angiotensin converting enzyme. FEBS. 2002; undefined:undefined.

[18] Letko M, Marzi A, Munster V. Functional assessment of cell entry and receptor usage for SARS-CoV-2 and other lineage B betacoronaviruses. Nat Microbiol. 2020

[19] Ou X, Liu Y, Lei X, et al. Characterization of spike glycoprotein of SARS-CoV-2 on virus entry and its immune cross-reactivity with SARS-CoV. Nat Commun. 2020;11(1):1620. Published 2020 Mar 27.

[20] Qiu ZF, Li TS, Fan HW, et al. Zhongguo Yi Xue Ke Xue Yuan Xue Bao. 2003;25(5):525-528.

[21] Shin HS, Kim Y, Kim G, et al. Immune Responses to Middle East Respiratory Syndrome Coronavirus During the Acute and Convalescent Phases of Human Infection. Clin Infect Dis. 2019;68(6):984-992.

[22] Zhou G, Zhao Q. Perspectives on therapeutic neutralizing antibodies against the Novel Coronavirus SARS-CoV-2. Int J Biol Sci. 2020;16(10):1718-1723. Published 2020 Mar 15.

[23] Ganji A, Farahani I, Khansarinejad B, et al. Increased expression of CD8 marker on T-cells in COVID-19 patients [published online ahead of print, 2020 Apr 13]. Blood Cells Mol Dis. 2020;83:102437.

[24] Hsueh, P.R., Huang, L.M., Chen, P.J., et al. 2004. Chronological evo- lution of IgM, IgA, IgG and neutralisation antibodies after infection with SARS- associated coronavirus. Clin. Microbiol. Infect. 10, 1062e1066. 
[25] Liu W, Fontanet A, Zhang PH, et al. Two-year prospective study of the humoral immune response of patients with severe acute respiratory syndrome. J Infect Dis. 2006;193(6):792-5.

[26] Guo L, Ren L, Yang S, et al. Profiling Early Humoral Response to Diagnose Novel Coronavirus Disease (COVID-19) [published online ahead of print, 2020 Mar 21]. Clin Infect Dis. 2020;ciaa310.

[27] Zhao J, Yuan Q, Wang H, et al. Antibody responses to SARS-CoV-2 in patients of novel coronavirus disease 2019 [published online ahead of print, 2020 Mar 28]. Clin Infect Dis. 2020;ciaa344.

[28] Cheung CY, et al. Cytokine responses in severe acute respiratory syndrome coronavirus-infected macrophages in vitro: possible relevance to pathogenesis. J Virol. 2005;79(12):7819-7826.

[29] Law HK, et al. Chemokine up-regulation in SARS-coronavirus-infected, monocyte-derived human dendritic cells. Blood. 2005;106(7):2366-2374.

[30] Yen YT, et al. Modeling the early events of severe acute respiratory syndrome coronavirus infection in vitro. J Virol. 2006;80(6):2684-2693.

[31] Lau SK, et al. Delayed induction of proinflammatory cytokines and suppression of innate antiviral response by the novel Middle East respiratory syndrome coronavirus: implications for pathogenesis and treatment. J Gen Virol. 2013;94(Pt 12):2679-2690.

[32] Chu H et al (2015) Middle East respiratory syndrome coronavirus efficiently infects human primary T lymphocytes and activates the extrinsic and intrinsic apoptosis pathways. J Infect Dis 213(6):904-14

[33] Tynell J, et al. Middle East respiratory syndrome coronavirus shows poor replication but significant induction of antiviral responses in human monocyte-derived macrophages and dendritic cells. J Gen Virol. 2016;97(2):344-355.

[34] Zhou J, et al. Active replication of Middle East respiratory syndrome coronavirus and aberrant induction of inflammatory cytokines and chemokines in human macrophages: implications for pathogenesis. J Infect Dis. 2014;209(9):1331-1342.

[35] Faure, E., Poissy, J., Goffard, A., Fournier, C., Kipnis, E., Titecat, M., Bortolotti, P., Martinez, L., Dubucquoi, S., Dessein, R., Gosset, P., Mathieu, D., Guery, B., 2014. Distinct immune response in two MERS-CoV-infected patients: can we go from bench to bedside? PloS One 9, e88716.

[36] Huang C, Wang Y, Li X, Ren L, Zhao J, Hu Y, et al. Clinical features of patients infected with 2019 novel coronavirus in Wuhan, China. Lancet. 2020;395:497-506.

[37] Mahallawi WH, Khabour OF, Zhang Q, Makhdoum HM, Suliman BA. MERS-CoV infection in humans is associated with a pro-infammatory T1 and T17 cytokine profle. Cytokine. 2018; 104:8-13.

[38] Wong CK, Lam CW, Wu AK, Ip WK, Lee NL, Chan IH, et al. Plasma infammatory cytokines and chemokines in severe acute respiratory syndrome. Clin Exp Immunol. 2004;136(1):95-103. 